\title{
TU/e emonownen

\section{Evaluation of angular scattering models for electron-neutral collisions in Monte Carlo simulations}

\section{Citation for published version (APA):}

Janssen, J. F. J., Pitchford, L. C., Hagelaar, G. J. M., \& van Dijk, J. (2016). Evaluation of angular scattering models for electron-neutral collisions in Monte Carlo simulations. Plasma Sources Science and Technology, 25(5), 1-12. [055028]. https://doi.org/10.1088/0963-0252/25/5/055026

DOI:

10.1088/0963-0252/25/5/055026

Document status and date:

Published: 22/09/2016

\section{Please check the document version of this publication:}

- A submitted manuscript is the version of the article upon submission and before peer-review. There can be important differences between the submitted version and the official published version of record. People interested in the research are advised to contact the author for the final version of the publication, or visit the $\mathrm{DOI}$ to the publisher's website.

- The final author version and the galley proof are versions of the publication after peer review.

- The final published version features the final layout of the paper including the volume, issue and page numbers.

Link to publication

\section{General rights}

Copyright and moral rights for the publications made accessible in the public portal are retained by the authors and/or other copyright owners and it is a condition of accessing publications that users recognise and abide by the legal requirements associated with these rights.

- Users may download and print one copy of any publication from the public portal for the purpose of private study or research.

- You may not further distribute the material or use it for any profit-making activity or commercial gain

- You may freely distribute the URL identifying the publication in the public portal.

If the publication is distributed under the terms of Article 25fa of the Dutch Copyright Act, indicated by the "Taverne" license above, please follow below link for the End User Agreement:

www.tue.nl/taverne

Take down policy

If you believe that this document breaches copyright please contact us at:

openaccess@tue.nl

providing details and we will investigate your claim. 


\title{
Evaluation of angular scattering models for electron-neutral collisions in Monte Carlo simulations
}

\author{
J F J Janssen ${ }^{1}$, L C Pitchford ${ }^{2}$, G J M Hagelaar ${ }^{2}$ and J van Dijk ${ }^{1}$ \\ 1 Department of Applied Physics, Eindhoven University of Technology, PO Box 513, \\ 5600 MB Eindhoven, The Netherlands \\ ${ }^{2}$ LAPLACE (Laboratoire Plasma et Conversion d'Energie), Universit de Toulouse, CNRS, INPT, \\ UPS; 118 route de Narbonne, F-31062 Toulouse, France \\ E-mail: j.f.j.janssen@tue.nl
}

Received 25 April 2016, revised 25 July 2016

Accepted for publication 19 August 2016

Published 22 September 2016

\begin{abstract}
In Monte Carlo simulations of electron transport through a neutral background gas, simplifying assumptions related to the shape of the angular distribution of electron-neutral scattering cross sections are usually made. This is mainly because full sets of differential scattering cross sections are rarely available. In this work simple models for angular scattering are compared to results from the recent quantum calculations of Zatsarinny and Bartschat for differential scattering cross sections (DCS's) from zero to $200 \mathrm{eV}$ in argon. These simple models represent in various ways an approach to forward scattering with increasing electron energy. The simple models are then used in Monte Carlo simulations of range, straggling, and backscatter of electrons emitted from a surface into a volume filled with a neutral gas. It is shown that the assumptions of isotropic elastic scattering and of forward scattering for the inelastic collision process yield results within a few percent of those calculated using the DCS's of Zatsarinny and Bartschat. The quantities which were held constant in these comparisons are the elastic momentum transfer and total inelastic cross sections.
\end{abstract}

Keywords: electron scattering, angular distribution, differential cross section, Monte Carlo

(Some figures may appear in colour only in the online journal)

\section{Introduction}

In Monte Carlo simulations of electron transport in low temperature plasmas, it is commonly assumed that the elastic momentum transfer cross section and the total cross sections for the inelastic scattering processes and for ionization provide sufficient information about electron interactions with the neutral background gas. The approach to forward scattering with increasing energy in electron-neutral collisions is usually not taken into account although some authors have done so by using simplified models for the angular scattering based on classical theory using a screened Coulomb potential or purely empirical formulae (see, for example, Boeuf \& Marode [1]; Kushner [2]; Surendra et al [3]; Belenguer \& Pitchford [4]). The absence of detailed knowledge about the differential scattering cross sections (DCS) for electron-neutral collisions generally precludes more precise calculations.

Previous work suggests that the additional information about the DCS's is not very important in electron swarm conditions (low degree of excitation and ionization, uniform field [5]) except for high values of reduced electric field strength, $\mathrm{E} / \mathrm{N}$, the ratio of the electric field strength to the neutral density or when high precision is required. See for example, discussions in Haddad et al [6]; Phelps and Pitchford [7]; Thomas \& Thomas [8]; Reid [9]; Kunhardt \& Tseng [10], Stojanovic and Petrovic [11]; among others. The conclusion from this literature is that the elastic momentum transfer cross section and total cross sections for excitation and ionization provide sufficient information for calculations of reasonably accurate swarm parameters up to E/N values of some $1000 \mathrm{Td}$. 
Note that assumptions related to the exact treatment of ionization processes are also important at high values of E/N. For example, assumptions related to growth renormalization [12, p 724, 725, 729] and energy sharing in ionization can impact the swarm parameters significantly $[7,13]$. In this work we are mainly interested in effects of anisotropy on parameters that are more sensitive to the shape of the DCS at small scattering angles. For that purpose a special test case is developed where the impact on the range and straggling of an electron beam is considered.

Recently Zatsarinny and Bartschat (ZB) calculated the DCS's for elastic and a number of inelastic processes in argon for electron energies ranging from threshold to $200 \mathrm{eV}$ [14]. These results are available through the LXCat website [15]. These calculated cross sections are in excellent agreement with recent high-precision measurements (Allan et al [16]) Thus, these calculations provide an opportunity to benchmark assumptions commonly made about the influence of anisotropic scattering in Monte Carlo simulations in conditions where the electron energy distribution function is not in equilibrium with the local value of $\mathrm{E} / \mathrm{N}$. The work presented in this paper is a step towards this objective.

In section 2 of the following, several models for angular scattering are described, and the extent to which these models accurately represent the approach to forward scattering with increasing incident electron energy is evaluated by comparing with the DCS's from Zatsarinny and Bartschat for elastic scattering and for excitation of the upper resonance level, $4 s^{\prime}[1 / 2]_{1}$, with a threshold at $11.828 \mathrm{eV}$. The DCS for this process is representative of most of the other allowed transitions. In section 3, we present results using the different models for anisotropy in Monte Carlo simulations of range, straggling and backscatter of electrons injected from a surface with a given energy into a background gas. In section 4, conclusions and a discussion about the relevance of this work to more general discharge situations are presented.

\section{Differential scattering cross sections}

The total cross section, $Q_{t}(\epsilon)$, is defined by integrating the DCS over all scattering directions $\Omega$. By assuming azimuthal symmetry the scattering directions can be calculated from $\mathrm{d} \Omega=2 \pi \sin \chi \mathrm{d} \chi$. The relation between the cross section and the DCS, $\frac{\mathrm{d} \sigma(\epsilon, \chi)}{\mathrm{d} \Omega}$, is then given by

$$
Q_{t}(\epsilon)=2 \pi \int \frac{\mathrm{d} \sigma(\epsilon, \chi)}{\mathrm{d} \Omega} \sin \chi \mathrm{d} \chi,
$$

with $\epsilon$ the energy and $\chi$ the angle between the velocity vectors of the incident particle before and after the collision. The angular distribution, $I(\epsilon, \chi)$, can be obtained by normalizing the DCS with the total cross section

$I(\epsilon, \chi)=\frac{1}{Q_{t}(\epsilon)} \frac{\mathrm{d} \sigma(\epsilon, \chi)}{\mathrm{d} \Omega} \leftrightarrow \leftrightarrow \frac{\mathrm{d} \sigma(\epsilon, \chi)}{\mathrm{d} \Omega}=Q_{t}(\epsilon) I(\epsilon, \chi)$.

A consequence of this definition is that the angular distribution has the property

$$
2 \pi \int I(\epsilon, \chi) \sin \chi \mathrm{d} \chi=1
$$

Equation (2) is a convenient formulation for our purposes because the shape of the cross section can be changed while keeping the total cross section, $Q_{t}$, constant. This is an important consideration when comparing results using different scattering models for the inelastic cross sections. For elastic collisions the constant quantity should be the elastic momentum transfer cross section so as to maintain the same momentum and energy loss in elastic scattering. This is easily accomplished in Monte Carlo simulations by setting the total elastic cross section equal to the momentum transfer cross section and thereafter assuming isotropic elastic scattering. The momentum transfer cross section $Q_{m}$ can be calculated from

$$
Q_{m}(\epsilon)=Q_{t}(\epsilon) 2 \pi \int I(\epsilon, \chi)(1-\cos \chi) \sin \chi \mathrm{d} \chi .
$$

Three types of analytical models for the angular distribution $I(\epsilon, \chi)$ will now be discussed. The first is derived from classical theory screened Coulomb scattering, and the second is from the Born approximation for inelastic scattering. These are all dependent on a single parameter. Thirdly, purely empirical formulae have also been proposed to describe the approach to forward scattering and these will also be briefly discussed.

In all cases, for numerical convenience in Monte Carlo simulations these analytical models are kept simple. In that case it is possible to obtain an analytical expression for the scattering angle in terms of a random number $R$ by inverting

$$
R=\frac{2 \pi \int_{0}^{\chi} I\left(\epsilon, \chi^{\prime}\right) \sin \chi^{\prime} \mathrm{d} \chi^{\prime}}{2 \pi \int_{0}^{\pi} I(\epsilon, \chi) \sin \chi \mathrm{d} \chi}
$$

The denominator in this expression is equal to one.

\subsection{Elastic screened Coulomb scattering}

Expressions for the angular distribution for elastic scattering have been derived for screened Coulomb scattering and presented previously in the low temperature plasma literature as discussed in [4, 17, 18].

Belenguer \& Pitchford [4] based their work on the screened Rutherford formula for differential scattering cross section given by Strickland et al [19] who express the angular distribution as,

$$
I(\epsilon, \chi)=\frac{\eta(\eta+1)}{\pi(2 \eta+1-\cos \chi)^{2}},
$$

where $\eta$ is the Coulomb screening parameter. According to Mott, the screening parameter can be calculated theoretically as $[20, \mathrm{p} 463]^{3}$

${ }^{3}$ Mott uses $\eta=\alpha^{2}=\left(0.565 Z^{1 / 3} s \frac{2 \pi e^{2}}{h v}\right)^{2}=0.565^{2} Z^{2 / 3} s^{2} \frac{2 \pi^{2} e^{4} m_{e}}{h^{2} \epsilon}$ with $s$ dimen-
sionless. Note that a factor $\frac{1}{\left(4 \pi \epsilon_{0}\right)^{2}}$ is required to convert to SI units. Mott uses $s=0.66$ to get agreement with the high energy limit of the Thomas-Fermi function. Additionally Mott mentions that in order to get agreement with the DCS from Hartree-Fock calculations for $Z>10$ the values cover the interval between $0.46 \leqslant s \leqslant 0.68$. 


$$
\eta=\frac{1.89 \mathrm{eV} Z^{2 / 3}}{\epsilon}
$$

with $Z$ the atomic number of the colliding particle and $\epsilon$ the energy. Strickland et al refer to Jacob [21] for the screening parameter, who gives an expression similar to equation (7) but with a prefactor of 10.9. The differences in the prefactor are not critical for the discussion here. Let us simply point out that several previous works have used the larger prefactor ([22, p 70] [23, p 34] [24, p 610]). Note that a higher prefactor causes the DCS to change from an isotropic to a forward directed distribution at a much higher energy.

The ratio of elastic momentum transfer to total elastic cross section, $Q_{m} / Q_{t}$, from the data compiled by Hayashi [25] in argon for energies up to $10 \mathrm{keV}$ is not consistent with screened Coulomb scattering with a screening parameter depending on $1 / \epsilon$. In an attempt to improve the accuracy of the angular distribution in equation (6), Belenguer and Pitchford let $\eta$ be a fitting function determined by matching to the Hayashi data for $Q_{m} / Q_{t}$. Although there is no reason to suppose that an angular distribution yielding a good representation of $Q_{m} / Q_{t}$ will also represent well the forward peak. Nevertheless, we use this ratio to fix $\eta$ because these data are sometimes available for elastic scattering of electrons and atoms or simple molecules whereas there are very few other data related to the angular distributions.

A similar approach was taken by Okhrimovskyy et al [17] who proposed a generalized expression for the angular distribution for screened Coulomb scattering:

$$
I(\epsilon, \chi)=\frac{1}{4 \pi} \frac{1-\xi^{2}(\epsilon)}{(1-\xi(\epsilon) \cos \chi)^{2}},
$$

where $\xi(\epsilon)$ is a fitting function. Equation (8) reduces to the angular distribution for conventional screened Coulomb scattering when $[17,18]$

$$
\xi=\frac{4 \epsilon / E_{\mathrm{H}}}{1+4 \epsilon / E_{\mathrm{H}}}
$$

with $E_{\mathrm{H}}$ representing one Hartree. Equations (6) and (8) are identical for $\xi=1 /(2 \eta+1)$ and so the screening length in equation (6) can be formally identified as

$$
\eta=\frac{E_{\mathrm{H}}}{8 \epsilon} \approx \frac{3.4 \mathrm{eV}}{\epsilon}
$$

This expression differs again from equation (7) in the prefactor but also by the lack of dependence on $Z$. Note that this relation is purely formal because $\eta$ can no longer be identified with the physical concept of a screening length for the Coulomb potential. We will henceforth not use the term screening length, but will retain the symbol $\eta$ and allow it to depend more generally on energy. As mentioned above, an alternate approach for estimating the energy dependence of the angular distribution is to choose $\eta$ or $\xi$ so that the ratio $Q_{m} / Q_{t}$ is well represented by equation (6) or (8), respectively. However, in order to use this method, both $Q_{m}$ and $Q_{t}$ must be known. The ratio can be calculated analytically from

$$
\frac{Q_{m}}{Q_{t}(\epsilon)}=2 \eta(\eta+1) \ln \left(1+\frac{1}{\eta}\right)-2 \eta,
$$

where $-\infty<\eta<-1$ for $\frac{Q_{m}}{Q_{t}(\epsilon)}>1$ and $0<\eta<\infty$ for $\frac{Q_{m}}{Q_{t}(\epsilon)}<1$.

Okhrimovskyy et al provide estimates of $\xi(\epsilon)$ (and hence $\eta$ ) for $\mathrm{Ar}, \mathrm{N}_{2}$ and $\mathrm{CH}_{4}$ based on the data compiled by Hayashi for the ratio $Q_{m} / Q_{t}$. As noted by Okhrimovskyy, a form for the angular dependence based on a screened Coulomb potential is not expected to be valid for polar molecules or other systems without spherical symmetry. Note, too, that many people use the formula for the scattering angle derived by Okhrimovskyy from the theory of screened Coulomb scattering for both elastic and inelastic scattering whereas inelastic scattering tends to be more peaked in the forward direction for a given energy (see for example, [20, p 483] or the data from Zatsarinny and Bartschat on LXCat [15]).

Scattering angles can be related to a random number via

$$
\cos \chi=\frac{\eta+1-R(1+2 \eta)}{\eta+1-R} .
$$

\subsection{Inelastic scattering}

An expression for the angular distribution of inelastic scattering can be derived from Mott et al [20] and Massey et al [26]. Interestingly this formula appears to be identical to an empirical formula suggested by Surendra. Surendra [3] used this formula for the elastic and inelastic scattering cross section of electrons and atoms. Surendra's expression is given by

$I(\epsilon, \chi)=\frac{\epsilon / \mathrm{eV}}{4 \pi\left[1+\epsilon / \mathrm{eV} \sin ^{2}\left(\frac{\chi}{2}\right)\right] \ln (1+\epsilon / \mathrm{eV})}$,

with $\mathrm{eV}$ used to obtain a dimensionless expression. Okhrimovskyy [17] and Khrabrov [18] note that this expression does not reduce to the correct limit for screened Coulomb interactions at high energies. Additionally Okhrimovskyy observed that the energy normalization is arbitrary (for example $1 \mathrm{eV}$ or 1 Hartree) and has no scientific background.

Based on the work of Mott et al [20, p 477, 493] and Massey et al [26, p 437] an expression for excitation can be obtained from

$$
I(K) K \mathrm{~d} K \propto \frac{1}{K^{2}}
$$

with

$$
K^{2}=k_{0}^{2}+k_{n}^{2}-2 k_{0} k_{n} \cos \chi,
$$

representing the momentum change of the scattered electron [20, p 476]. The relation between momentum and energy is given by

$$
\epsilon_{k}=\frac{\hbar^{2} k^{2}}{2 m}
$$

This approximation uses the expansion $\exp (\mathrm{i} K z) \doteq 1+\mathrm{i} K z$ and is therefore not valid when the momentum exchange is large. Note that the momentum exchange is largest for backscattering. The formula is therefore most accurate for small 
angles. Additionally the assumption is made that the relative energy transfer in collisions is small. Substituting this result in the DCS and changing variables from $K$ to $\chi$ gives

$$
I(\epsilon, \chi) \sin \chi \mathrm{d} \chi \propto \frac{\sin \chi \mathrm{d} \chi}{K^{2}} .
$$

After normalization the DCS is given by [27] $]^{4}$

$$
I(\epsilon, \chi)=\frac{\beta}{2 \pi \ln \left(\frac{1+\beta}{1-\beta}\right)\left(1+\beta^{2}-2 \beta \cos \chi\right)},
$$

with

$$
\beta=\sqrt{1-\frac{\epsilon_{n 0}}{\epsilon_{n}}},
$$

where $\epsilon_{n 0}$ is the atomic transition energy and $\epsilon_{n}$ the initial energy of the electron. By applying the substitution $\epsilon / \mathrm{eV}=\frac{4 \beta}{(\beta-1)^{2}}$ it can be shown that the inelastic formula is identical to Surendra's formula.

Alternatively, the parameter $\beta$ can be determined from $Q_{t}$ and $Q_{m}$ as was done by Surendra [3]. In terms of the inelastic formula this ratio can be expressed as

$$
\frac{Q_{m}}{Q_{t}(\epsilon)}=1-\frac{1+\beta^{2}}{2 \beta}-\frac{1}{\ln \left(\frac{1+\beta}{1-\beta}\right)} .
$$

The momentum transfer cross section for inelastic processes are rarely available and so this approach is of limited utility. For this DCS the scattering angle is related to a random number via

$$
\cos \chi=\frac{1+\beta^{2}-(1+\beta)^{2} \exp (R)}{2 \beta} .
$$

\subsection{Empirical formulae}

In this section various empirical formulae and the corresponding relations between scattering angle and random number are discussed. The considered DCS are isotropic scattering, forward scattering and empirical formulae given by Kushner, Longo et al, Belenguer et al as well as another convenient formula representing a best fit to the calculations of Zatsarinny and Bartschat.

Isotropic scattering is often used to describe the angular behavior of elastic collisions. The angular distribution is constant

$$
I(\epsilon, \chi)=\frac{1}{4 \pi}, \quad \cos \chi=1-2 R .
$$

The ratio of the momentum transfer cross section and the total cross section reveals that for an isotropic DCS the momentum transfer cross section is equal to the total cross section $\left(Q_{t}=Q_{m}\right)$. Inelastic processes are sometimes modelled

\footnotetext{
${ }^{4}$ Based on the theory of the generalized oscillator strength [20, p 478] [28] the same angular distribution is obtained.
}

with forward scattering. In that case the electron continues in the same direction without being scattered

$$
I(\epsilon, \chi)=\frac{\delta(\chi)}{2 \pi \sin \chi}, \quad \cos \chi=1 .
$$

Longo and Capitelli [29] propose another way of using $Q_{t}$ and $Q_{m}$ for elastic scattering, when both are available, in Monte Carlo codes. Their idea is to model the DCS as the sum of two terms one representing isotropic scattering in the forward direction $(\chi \leqslant \pi / 2)$ and the other isotropic scattering in the backward direction $(\chi \geqslant \pi / 2)$. The magnitudes of each of the two terms are adjusted for consistency with known values of $Q_{t}$ and $Q_{m}$. Kushner [2] introduced an empirical expression for forward scattering which is given by

$$
I(\epsilon, \chi)=\frac{n+2}{8 \pi} \cos ^{n}\left(\frac{\chi}{2}\right),
$$

with $n$ an energy dependent fitting parameter. Kushner suggests to set $n=3$ when no experimental data are available. This parameterization contains no dependence on the initial kinetic energy and thus will not be able to reproduce the DCS for a large spectrum of energies. In this case the connection between the scattering angle and a random number is given by

$$
\cos \chi=2(1-R)^{2 /(n+2)}-1 .
$$

Another empirical formula has been suggested by Belenguer and Pitchford [4] and is given by

$$
I(\epsilon, \chi)=I_{0}(\epsilon)+I_{1}(\epsilon) \cos \chi+I_{2}(\epsilon) \cos ^{2} \chi .
$$

This method requires $Q_{t}(\epsilon), Q_{m}(\epsilon)$ and $Q_{v}(\epsilon)$ (the viscosity cross section, obtained by multiplying the DCS by $\left(1-\cos ^{2} \chi\right)$ and integrating over all angles) to obtain the values of $I_{0}(\epsilon)$, $I_{1}(\epsilon)$ and $I_{2}(\epsilon)$. The calculations reported by Belenguer and Pitchford used data from Hayashi [25] for these quantities for elastic scattering. It is also possible to combine multiple processes in a single DCS

$$
I(\epsilon, \chi)=\frac{C}{4 \pi}+(1-C) \frac{\eta(\eta+1)}{\pi(2 \eta+1-\cos \chi)^{2}} .
$$

This functional form represents the sum of an isotropic component and an anisotropic component represented by the screened Coulomb form. In that case the energy dependent parameters $C$ and $\eta$ can be derived from direct fits or by imposing two constraints. In this work a direct fit using

$$
C(\epsilon)=\frac{\epsilon}{p_{1}}+p_{2},
$$

and

$$
\eta(\epsilon)=\left(\frac{d_{1}}{\epsilon}\right)^{d_{2}},
$$

was used with $p_{i}$ and $d_{i}$ fit parameters.

\subsection{Comparison to the ab initio DCS}

This section contains an evaluation of the theoretical estimates of the DCS's given in the previous sections. Only the 


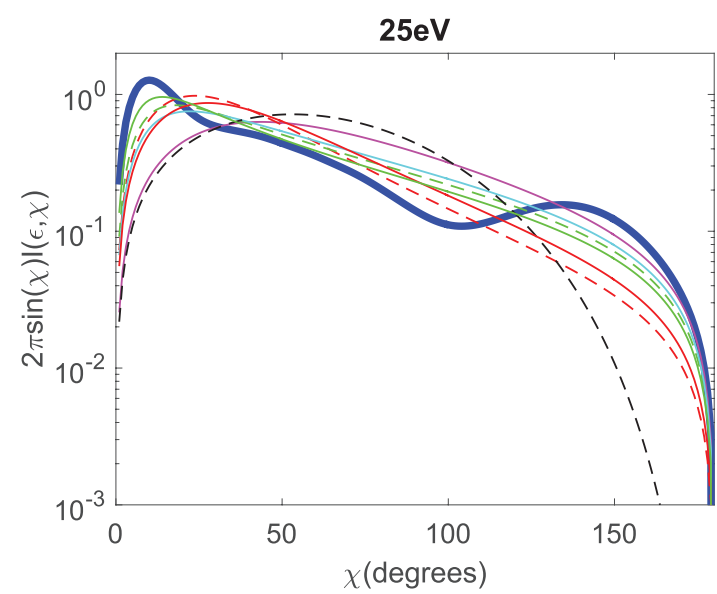

(a)

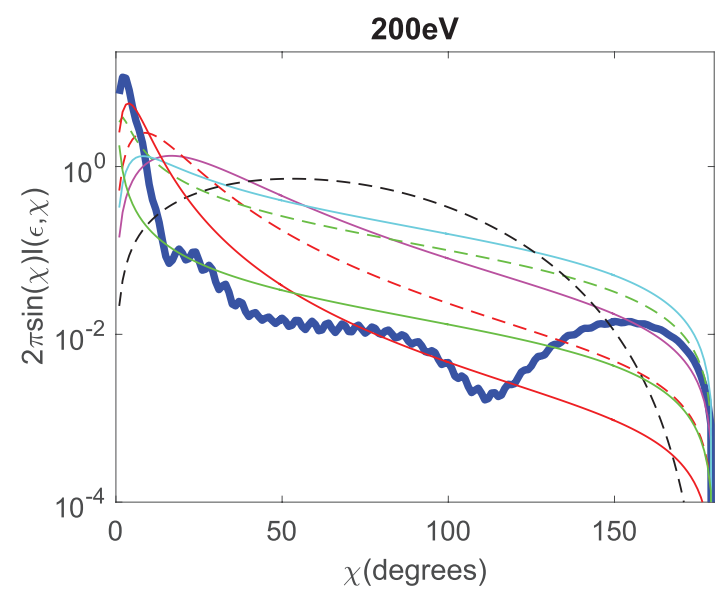

(c)

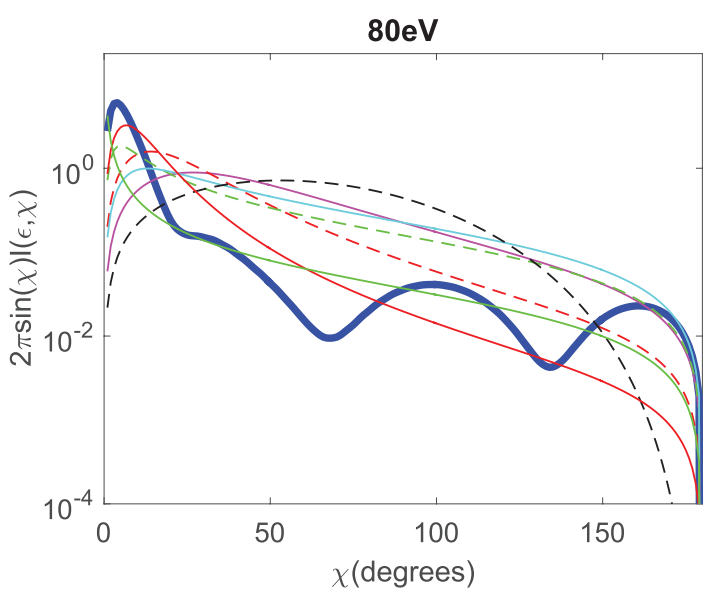

(b)

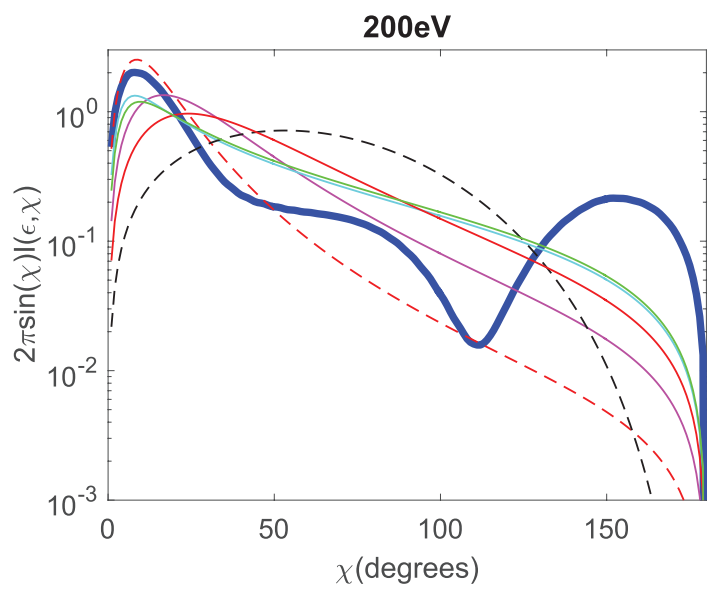

(d)

Figure 1. The DCS for various angular distributions and initial kinetic energies. The inelastic process refers to excitations towards $4 s^{\prime}[1 / 2]_{1}$. The labels are explained in table 1. Legend: ZB (blue); Mott (magenta); SC (dashed line, red); SCI (dashed line, green); Surendra (cyan); Kushner (dashed line, black); SC $Q_{m} / Q_{t}$ (red); SCI $Q_{m} / Q_{t}$ (green). (a) Inelastic DCS at $25 \mathrm{eV}$. (b) Inelastic DCS at $80 \mathrm{eV}$. (c) Inelastic DCS at $200 \mathrm{eV}$. (d) Elastic DCS at $200 \mathrm{eV}$.

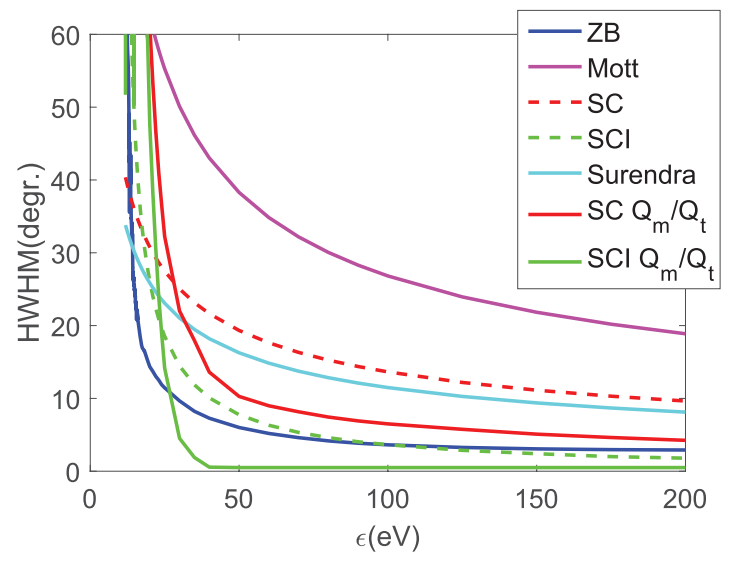

(a)

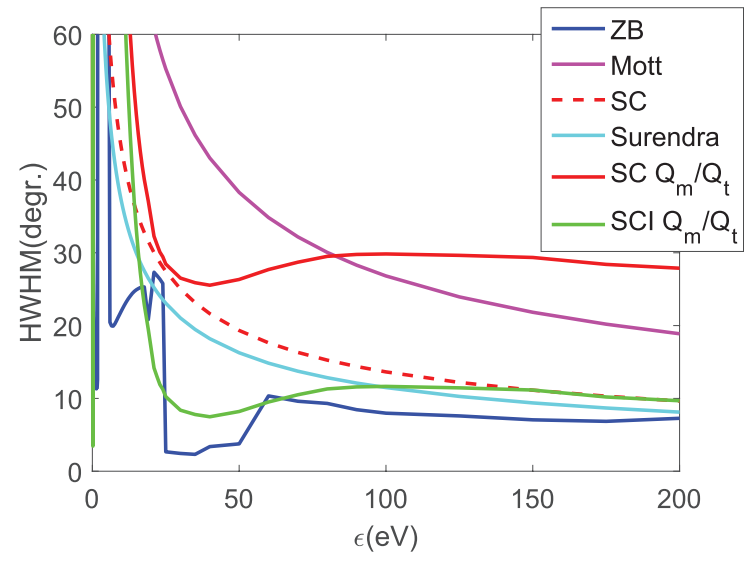

(b)

Figure 2. The HWHM for various angular distributions. The labels are explained in table 1. (a) Excitation from the ground state to $4 \mathrm{~s}^{\prime}[1 / 2]_{1}$. (b) Elastic collisions.

results for the excitation from the ground state to $4 \mathrm{~s}^{\prime}[1 / 2]_{1}$ are shown since the results of the other optically allowed transitions are very similar. The results for incident energies of 25 , 80 and $200 \mathrm{eV}$ for the inelastic process and the $200 \mathrm{eV}$ for the elastic process are shown in figures 1(a)-(d). The half-widthat-half-maximum (HWHM) for the angular distribution of both processes is shown in figures 2(a) and (b). The labels in the figures are explained in table 1 . 
Table 1. Definition of the labels that have been used in the figures 1(a)-2(b).

\begin{tabular}{ll}
\hline Label & Explanation \\
\hline $\mathrm{ZB}$ & Quantum mechanical calculation of DCS data from Zatsarinny and Bartschat \\
$\mathrm{Mott}$ & Elastic screened Coulomb (6) with $\eta$ from (7) for the screening parameter \\
$\mathrm{SC}$ & Elastic screened Coulomb (6) with $\eta$ from (10) \\
$\mathrm{SC} Q_{m} / Q_{t}$ & Elastic screened Coulomb (6) with $\eta$ from the ratio $Q_{m} / Q_{t}(11)$ using the data from ZB for elastic scattering \\
$\mathrm{SCI}$ & Inelastic screened Coulomb (18) with $\beta$ from (19) \\
$\mathrm{SCI} Q_{m} / Q_{t}$ & Inelastic screened Coulomb (18) with $\beta$ from the ratio $Q_{m} / Q_{t}(20)$ using the data from ZB for inelastic scattering \\
$\mathrm{Surendra}$ & Empirical formula proposed by Surendra (13) for elastic and inelastic processes. \\
Kushner & Empirical formula (24) suggested by Kushner with $n=3$. \\
$\mathrm{CUS}$ & Direct fit using (27) for optically allowed inelastic transitions with $C$ given by (28) and $\eta$ given by (29). \\
\hline
\end{tabular}

The excitation process is not well described by any of the generalized models. The best results are obtained by using the ratio $\frac{Q_{m}}{Q_{t}(\epsilon)}$ to estimate $\eta$ in the elastic screened Coulomb formula. This approach roughly describes the increased probability at small angles and the subsequent decay for larger angles. The HWHM of the angular distribution is also roughly described accurately by this approach. However the oscillations at larger angles found by Zatsarinny and Bartschat are of course not reproduced. Especially the probability for backscattering is underestimated. The inelastic formula with the analytical parameterization gives the best agreement with the HWHM derived from the data of Zatsarinny and Bartschat. However the angular distribution does not describe the peak well and significantly overestimates the probability for large angle scattering. Similarly a fit of $Q_{m} / Q_{t}$ using the inelastic formula underestimates the peak and its width while it describes the large angle scattering relatively well. The remaining methods describe neither the peak nor the width accurately.

For elastic collisions the theoretical expressions perform better. The model SC which uses the parameterization given by Okhrimovskyy performs well for the probability at small angles. The HWHM of the angular distribution approaches the correct value for energies above $60 \mathrm{eV}$. The decreasing trend for the probability at larger angles is also reproduced. However the oscillations in the angular distribution which lead to a considerably larger backscattering probability are not captured by the model. Interestingly the model proposed by Surendra and the SCI fit of $Q_{m} / Q_{t}$ also produces a reasonable agreement with the scattering probability at small angles. The backscattering probability is slightly underestimated. Additionally the width of the angular distribution follows a similar behavior as the SC model for energies above $100 \mathrm{eV}$. In comparison to the SC model the decaying trend for the probability of scattering is much smaller. This trend is more in agreement with the large angle behavior of Zatsarinny and Bartschat, but still cannot capture the various oscillations.

In summary, the simple models aim only to capture the approach to forward scattering with increasing energy. They are not intended to reproduce the structure at low energy or the backscatter at intermediate energies. The inelastic processes are approximated most accurately with the SC $Q_{m} / Q_{t}$ model where 'accuracy' is evaluated by comparing the probability at small angles and the HWHM of the forward peak in the calculations. This approximation still underestimates the scattering probability at small angles by a factor in the order of 2 at $200 \mathrm{eV}$. Additionally the width of the angular distribution is overestimated by this formula. The inelastic processes are therefore not accurately represented with the theoretical formulae. The elastic cross sections are most accurately represented by the SC model. Both the probability for small angles and the width of the distribution are described accurately. However the large angle scattering is underestimated.

\section{Quantification of anisotropy using Monte Carlo}

The effects of the different scattering models are investigated for Monte Carlo simulations of a very simple model system using the modelling platform PLASIMO [23, 30]: a $50-200 \mathrm{eV}$ electron beam injected into a background gas of simplified argon atoms. Electrons in this energy range occur in negative glows near the cathode in dc gas discharges and constitute perhaps the most basic system in which effects of anisotropic scattering are expected to show up. The simplified argon atoms that are considered can interact with an electron according to the following processes:

- An elastic process.

- An excitation process. The angular distribution is taken from the excitation from the ground state towards $4 s^{\prime}[1 / 2]_{1}$. The cross section of the inelastic process is scaled to vary the importance of the inelastic collisions.

This two-level system is sufficient for the purpose of comparing quantities calculated with different models for the angular scattering for both processes individually ${ }^{5}$. The scattering of the elastic process is approximated with two different models. These are isotropic scattering and the DCS calculated by Zatsarinny and Bartschat. Different scattering models are considered for the inelastic process.

The scale factors that are considered are based on the sum of all cross sections. These cross sections are given in figure 3 . The data for the excitation processes are obtained by integrating the DCS provided by Zatsarinny and Bartschat. The

\footnotetext{
${ }^{5}$ The data set provided by Zatsarinny and Bartschat does not contain the ionization process. An improved model can be made if the DCS for the ionization process is more accurately known. Additionally, when ionization processes are included other choices impact the model as well. One of these choices is the energy sharing ratio. In this work only the impact of the DCS on the range and straggling is evaluated.
} 


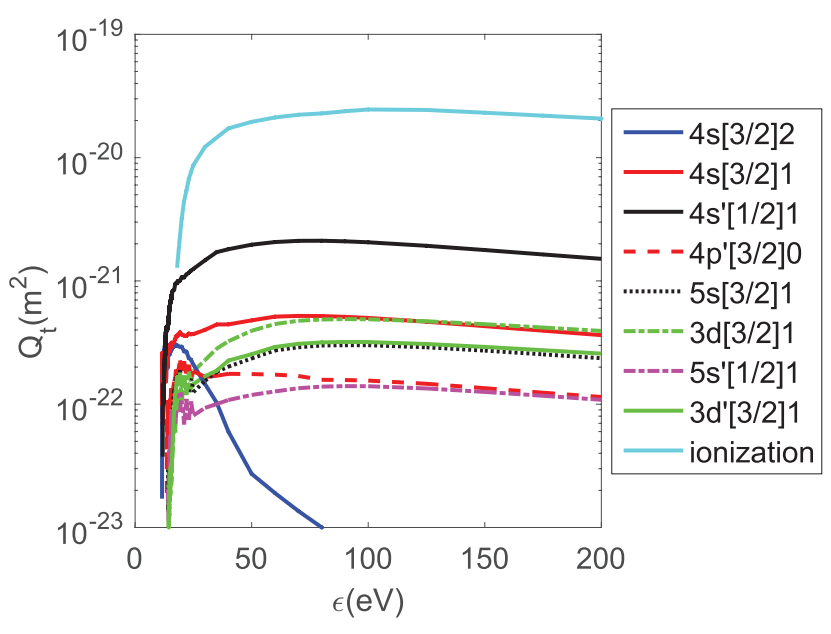

Figure 3. Cross sections obtained by integrating the DCS from [15]. The ionization cross section is taken from [14, 16, 31]. The excitation cross sections deviate slightly between both datasets.

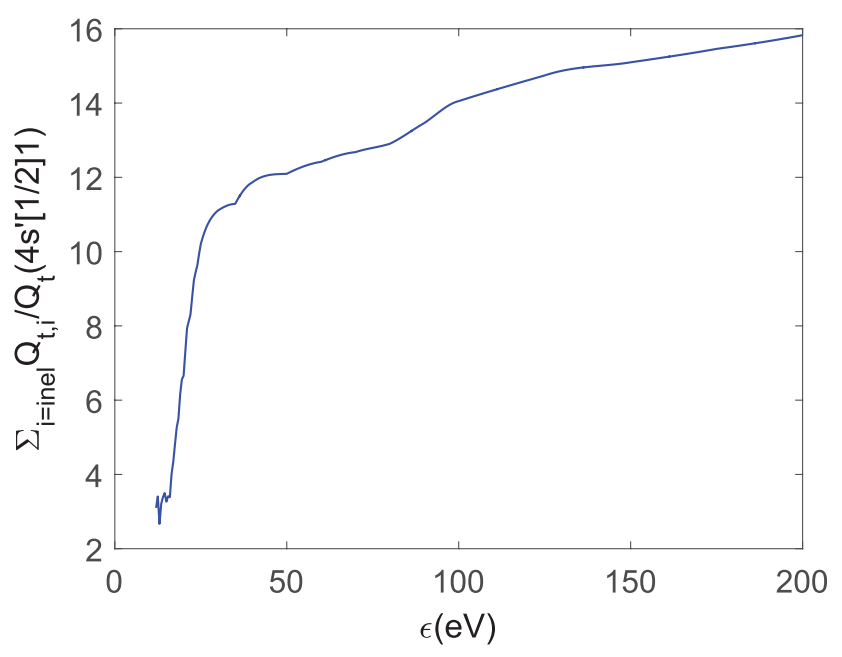

Figure 4. The ratio of the sum of all inelastic processes (including ionization) relative to the excitation process towards $4 s^{\prime}[1 / 2]_{1}$.

DCS for the ionization process was not calculated so the total scattering from other calculations of Zatsarinny and Bartschat is considered here in calculating the sum of the total cross sections $[14,16,31]$. The strongest excitation process is the transition towards $4 s^{\prime}[1 / 2]_{1}$. However the ionization process has an even larger cross section. In order to represent approximately the sum of all inelastic and ionization processes, the transition towards $4 s^{\prime}[1 / 2]_{1}$ should be scaled by a factor of about 10 as indicated in figure 4 . An overestimate of the importance of the inelastic DCS can be obtained by scaling the inelastic process with a factor of 100 . Both cases are investigated. The total cross sections and momentum transfer cross section for the elastic and inelastic processes considered are displayed in figure 5. For $\epsilon>50 \mathrm{eV}$ the elastic and the inelastic processes have approximately equal probabilities when isotropic scattering is used with $Q_{t}=Q_{m}$ for elastic scattering.

The simulated geometry consists of an infinite half-space $(z \leqslant 0)$. This plane represents a cathode. Electrons are emitted with a given energy from the center of the cathode at $r=0$ with a velocity directed normal to the surface. Electrons backscattered to the cathode are removed from the simulation. In

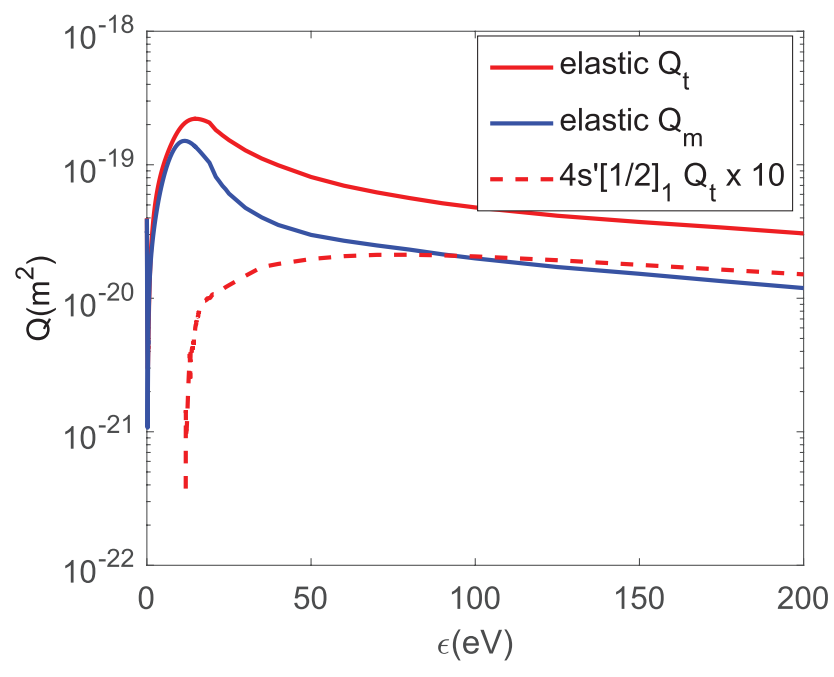

Figure 5. The total and momentum transfer cross section for the elastic and the inelastic $4 s^{\prime}[1 / 2]_{1}$ transition are shown. The inelastic cross sections are scaled by a factor of 10 .

this test the focus lies on the distribution of the inelastic scattering events. Electrons that do not have enough energy left for another excitation process are therefore removed. Two statistics related to this position are collected

- Range: It is defined as the average position in space where electrons are removed from the simulation, i.e. where their energy drops below the excitation threshold. These electrons will be referred to as 'stopped' electrons. The range can thus be calculated from

$$
R_{z}=\langle z\rangle=\int_{0}^{\infty} z P(r, z) \mathrm{d} V
$$

with $P(r, z)$ the probability density function of stopped electrons per volume $V$.

- Straggling: It is a measure of the spread in the range. It is defined as the standard deviation of the range and can be calculated from

$$
S_{z}=\left\langle\left(z-R_{z}\right)^{2}\right\rangle=\int_{0}^{\infty} z^{2} P(r, z) \mathrm{d} V-R_{z}^{2} .
$$

Analogous expressions can be derived for the radial direction. A total number of $10^{8}$ electrons is considered. The Monte Carlo code used for these simulations is described by Brok [23] The standard deviation of the mean (range) is then given by [32]

$$
S_{R}=\frac{S}{\sqrt{n}}=\frac{S}{10^{4}},
$$

with $R$ representing the range and $S$ the straggling. Similarly the standard deviation of the straggling is approximated with

$$
S_{S}=\frac{S}{\sqrt{2(n-1)}} \approx 7 \cdot 10^{-5} S .
$$

Since the straggling is of the same order of magnitude as the range the relative accuracy of both quantities is in the order of $10^{-4}$.

For an initial energy of $200 \mathrm{eV}$ and a scale factor of 10 the distribution of stopped electrons is shown for different 


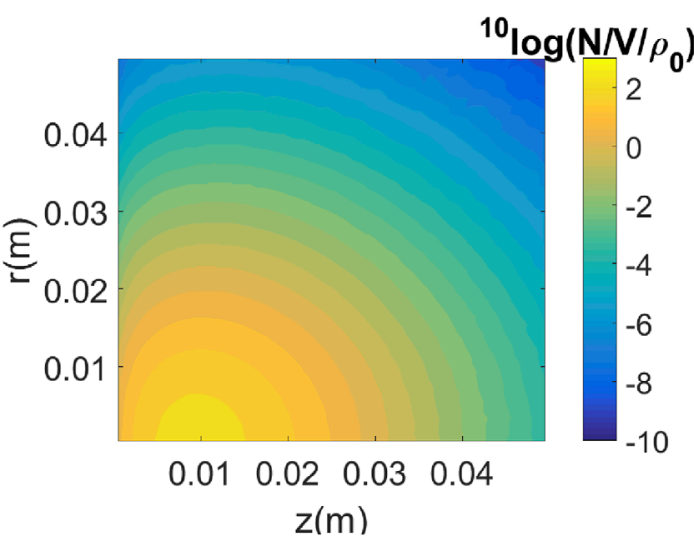

(a)

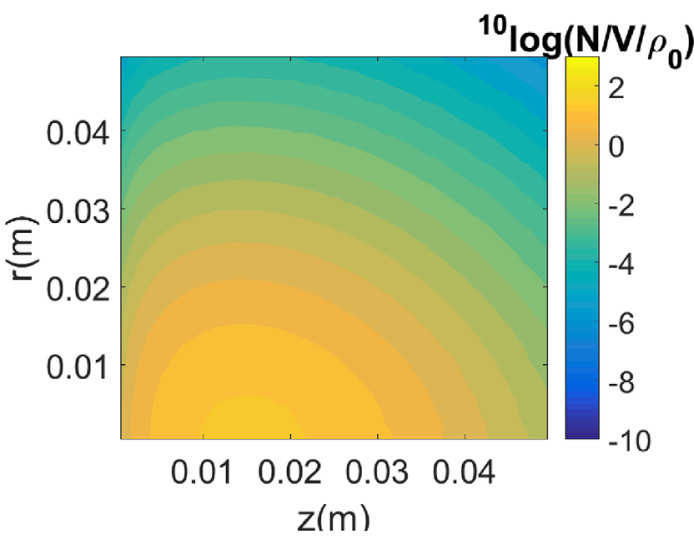

(c)

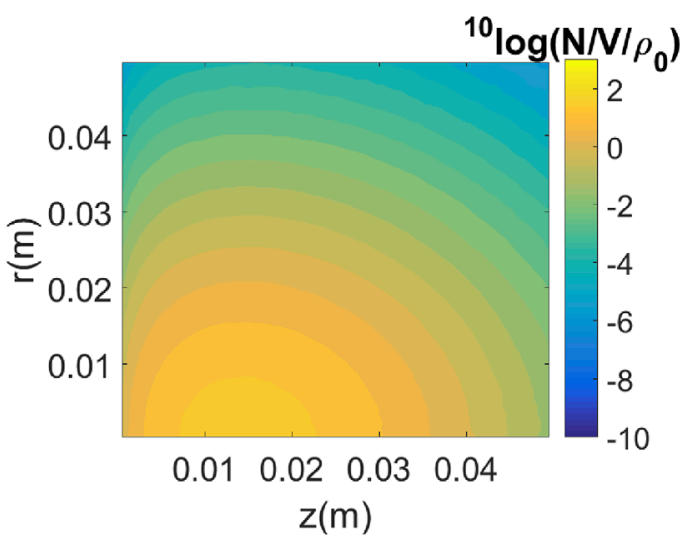

(e)

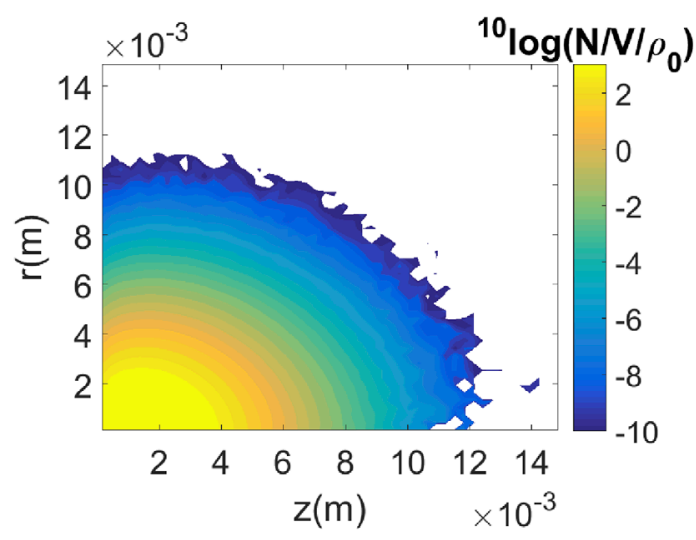

(b)

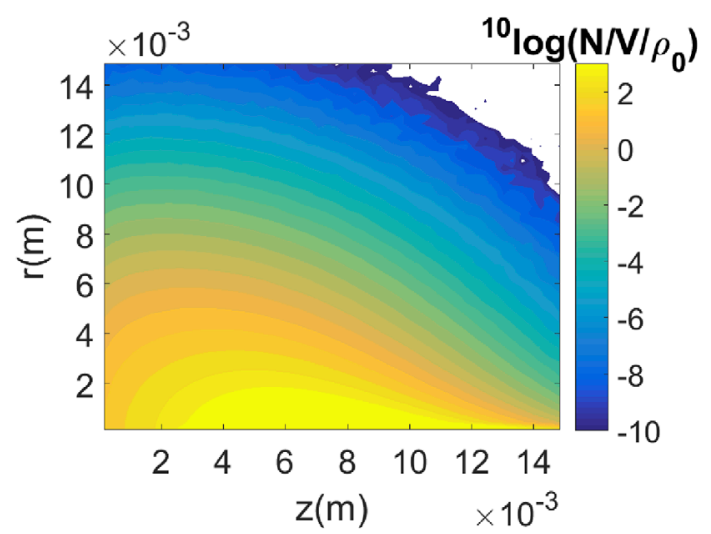

(d)

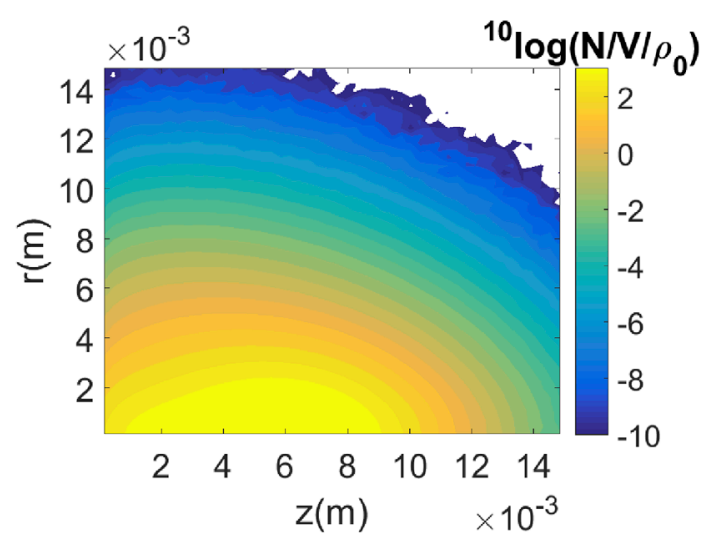

(f)

Figure 6. Spatial profile of stopped electrons expressed as a density by dividing the number of electrons removed by the local volume. The results are normalized by $\rho_{0}$ which is calculated as the total number of electrons divided by the displayed volume. An input energy of $200 \mathrm{eV}$ has been used in all cases. The scale factor for the inelastic transition is varied and the DCS of the elastic and inelastic DCS are varied. (a) Elastic and inelastic scattering: isotropic; scale factor: 10. (b) Elastic and inelastic scattering: isotropic; scale factor: 100.

(c) Elastic scattering: isotropic; Inelastic scattering: forward; scale factor: 10. (d) Elastic scattering: isotropic; Inelastic scattering: forward; scale factor: 100. (e) Elastic and inelastic scattering: ZB; scale factor: 10. (f) Elastic and inelastic scattering: ZB; scale factor: 100.

elastic and inelastic scattering models in figure 6(a), (c) and (e). The spatial profile of stopped electrons using the data from Zatsarinny and Bartschat is similar to the profile obtained using forward scattering (FOR). The calculated range using isotropic scattering (ISO) is significantly reduced in comparison to the other models. These differences can be explained by noting that the probabilities for an inelastic collision are in the order of $50 \%$ for $\epsilon>100 \mathrm{eV}$. For isotropic inelastic collisions all collisions are isotropic while for forward scattering only half of the collisions are isotropic. The differences between FOR and the ab initio results are much smaller since for $\epsilon>100 \mathrm{eV}$ the $a b$ initio results converge to 
Table 2. Results for a scale factor of 10 and an input energy of $200 \mathrm{eV}$.

\begin{tabular}{lcccccc}
\hline Simulation & $R_{z}(\mathrm{~m})$ & error $(\%)$ & $S_{z}(\mathrm{~m})$ & error $(\%)$ & $S_{x}(\mathrm{~m})$ & $B(\%)$ \\
\hline \multicolumn{7}{c}{ Isotropic elastic scattering with $Q_{m}$ from ZB } \\
\hline ZB & $1.98 \times 10^{-02}$ & 0.0 & $1.16 \times 10^{-02}$ & 0.0 & $1.50 \times 10^{-02}$ & 51.6 \\
CUS & $2.00 \times 10^{-02}$ & 1.3 & $1.18 \times 10^{-02}$ & 1.1 & $1.53 \times 10^{-02}$ & 51.1 \\
FOR & $2.03 \times 10^{-02}$ & 2.4 & $1.19 \times 10^{-02}$ & 2.3 & $1.53 \times 10^{-02}$ & 51.0 \\
ISO & $1.45 \times 10^{-02}$ & -26.7 & $8.74 \times 10^{-03}$ & -25.0 & $1.19 \times 10^{-02}$ & 65.1 \\
SC $Q_{m} / Q_{t}$ & $1.97 \times 10^{-02}$ & -0.5 & $1.16 \times 10^{-02}$ & -0.7 & $1.51 \times 10^{-02}$ & 51.4 \\
SC & $1.87 \times 10^{-02}$ & -5.3 & $1.10 \times 10^{-02}$ & -5.2 & $1.48 \times 10^{-02}$ & 52.6 \\
SCI & $1.79 \times 10^{-02}$ & -9.6 & $1.06 \times 10^{-02}$ & -8.9 & $1.43 \times 10^{-02}$ & 55.2 \\
\hline \multicolumn{7}{r}{ Anisotropic elastic scattering from ZB } \\
ZB & $2.01 \times 10^{-02}$ & 0.0 & $1.18 \times 10^{-02}$ & 0.0 & $1.51 \times 10^{-02}$ & 50.8 \\
CUS & $2.03 \times 10^{-02}$ & 1.3 & $1.19 \times 10^{-02}$ & 1.1 & $1.53 \times 10^{-02}$ & 50.4 \\
FOR & $2.06 \times 10^{-02}$ & 2.5 & $1.20 \times 10^{-02}$ & 2.3 & $1.54 \times 10^{-02}$ & 50.3 \\
ISO & $1.45 \times 10^{-02}$ & -27.5 & $8.77 \times 10^{-03}$ & -25.4 & $1.20 \times 10^{-02}$ & 64.7 \\
SC $Q_{m} / Q_{t}$ & $1.99 \times 10^{-02}$ & -0.6 & $1.17 \times 10^{-02}$ & -0.7 & $1.52 \times 10^{-02}$ & 50.6 \\
SC & $1.89 \times 10^{-02}$ & -5.8 & $1.11 \times 10^{-02}$ & -5.3 & $1.49 \times 10^{-02}$ & 51.9 \\
SCI & $1.80 \times 10^{-02}$ & -10.3 & $1.07 \times 10^{-02}$ & -9.1 & $1.43 \times 10^{-02}$ & 54.6 \\
\hline
\end{tabular}

Note: ' $R$ ' represents the range, ' $S$ ' the straggling and ' $B$ ' the electrons backscattered to the cathode. The top section shows the results by assuming an isotropic DCS for the electrons with the elastic $Q_{m}$ from ZB. The bottom section shows the results by using the elastic DCS calculated by ZB. The labels are defined in table 1.

the FOR model. The differences between FOR and the data from Zatsarinny and Bartschat are more pronounced on the axis since the probability of forward scattering decreases for lower energies in the $a b$ initio results. Further away from the axis the discrepancies between the two sets are smaller, since electrons that can reach these positions encountered at least one (random) large angle collision.

The data for all considered models is shown in table 2 . The table shows that the CUS model, given by equation (27), and the elastic screened Coulomb fit using SC $\frac{Q_{m}}{Q_{t}}$ provide an accurate description of the range and straggling. In comparison with the $a b$ initio data the relative errors for the axial range are in the order of $1 \%$. The elastic screened Coulomb model (SC) and the inelastic screened Coulomb model (SCI) are less accurate with relative errors ranging from 5 to $10 \%$. In the absence of any DCS or $Q_{m}$ data the CUS and SC $\frac{Q_{m}}{Q_{t}}$ models cannot be used. The best approximation in such a situation is the FOR model with a relative error of $2.4 \%$ in comparison to the $a b$ initio data. The backscattering for most models is predicted correctly within $2 \%$ of the data from Zatsarinny and Bartschat. The exceptions are the isotropic and SCI models which differ more from the baseline case $(\mathrm{ZB})$ than the other models do. Similar conclusions can be made when the DCS calculated by Zatsarinny and Bartschat is used for elastic collisions. In fact the results only differ up to $2 \%$ in comparison to the simulation that uses isotropic scattering for elastic collisions with the same momentum transfer cross section. The results confirm that the elastic collisions are approximated well with isotropic scattering. This is expected since figure 1(d) shows a DCS at $200 \mathrm{eV}$ that decays slowly as a function of scattering angle.

The influence of use of different models for anisotropic scattering can be emphasized by using a scaling factor of 100 for the inelastic process. These results are shown in figure 6(b), (d) and (f). The spatial profile of stopped electrons using the FOR model is still quite similar to the results of the $a b$ initio calculations. The main differences are visible on the axis. Due to the increased cross section of the inelastic collision the probability of an inelastic collision is larger than two times the probability of an elastic collision for $\epsilon>20 \mathrm{eV}$. As a result many electrons in the FOR model did not have a single elastic encounter. These electrons are still on the axis when their energy drops below the threshold energy. Another effect of the increased probability of the inelastic process is that the differences between the $a b$ initio results and the ISO model are much more pronounced in the spatial profile of stopped electrons.

The data for all considered models is shown in table 3. Again the CUS and SC $Q_{m} / Q_{t}$ model give the best reproduction of the axial range. The relative errors are $7.1 \%$ and $4.1 \%$ respectively. Even for these extreme conditions the FOR model produces a relative error of only $15.6 \%$. These errors are mainly caused by the fact that the $a b$ initio data contain a small probability for large angle scattering. This effect is more pronounced for a DCS at lower energies. The predictions for the axial range by the SC and SCI models are off by $24.7 \%$ and $36.1 \%$, respectively. Thus for an initial kinetic energy of $200 \mathrm{eV}$ the FOR model also requires no additional input and performs better. The backscattering is not well predicted with such large cross sections. Most models make an error in the order of $14-22 \%$. Larger errors are made for the ISO and the SCI models. The impact on the results induced by changing the elastic cross section to the calculated cross section by Zatsarinny and Bartschat now is a bit stronger. However the impact is restricted to about $5 \%$ and is therefore still relatively small.

Similar tests have been made for input energies of $50 \mathrm{eV}$. The results of these tests are shown in table 4. For a scale factor of 10 the results are similar to the ab initio results, since 
Table 3. Results for a scale factor of 100 and an input energy of $200 \mathrm{eV}$.

\begin{tabular}{llclclc}
\hline Simulation & $R_{z}(\mathrm{~m})$ & error $(\%)$ & $S_{z}(\mathrm{~m})$ & error $(\%)$ & $S_{x}(\mathrm{~m})$ & $B(\%)$ \\
\hline \multicolumn{5}{c}{ Isotropic elastic scattering with $Q_{m}$ from ZB } \\
\hline ZB & $5.01 \times 10^{-03}$ & 0.0 & $2.59 \times 10^{-03}$ & 0.0 & $2.27 \times 10^{-03}$ & 18.6 \\
CUS & $5.37 \times 10^{-03}$ & 7.1 & $2.59 \times 10^{-03}$ & 0.0 & $2.35 \times 10^{-03}$ & 14.9 \\
FOR & $5.79 \times 10^{-03}$ & 15.6 & $2.79 \times 10^{-03}$ & 7.8 & $2.18 \times 10^{-03}$ & 14.6 \\
ISO & $1.98 \times 10^{-03}$ & -60.5 & $1.26 \times 10^{-03}$ & -51.5 & $1.64 \times 10^{-03}$ & 58.6 \\
SC $Q_{m} / Q_{t}$ & $4.80 \times 10^{-03}$ & -4.1 & $2.41 \times 10^{-03}$ & -6.9 & $2.51 \times 10^{-03}$ & 16.0 \\
SC & $3.77 \times 10^{-03}$ & -24.7 & $2.11 \times 10^{-03}$ & -18.4 & $2.54 \times 10^{-03}$ & 22.0 \\
SCI & $3.20 \times 10^{-03}$ & -36.1 & $1.93 \times 10^{-03}$ & -25.4 & $2.34 \times 10^{-03}$ & 33.3 \\
\hline \multicolumn{7}{c}{ Anisotropic elastic scattering from ZB } \\
ZB & $5.13 \times 10^{-03}$ & 0.0 & $2.54 \times 10^{-03}$ & 0.0 & $2.25 \times 10^{-03}$ & 19.1 \\
CUS & $5.51 \times 10^{-03}$ & 7.3 & $2.52 \times 10^{-03}$ & -0.9 & $2.33 \times 10^{-03}$ & 15.4 \\
FOR & $5.99 \times 10^{-03}$ & 16.8 & $2.67 \times 10^{-03}$ & 4.8 & $2.14 \times 10^{-03}$ & 15.5 \\
ISO & $1.98 \times 10^{-03}$ & -61.4 & $1.26 \times 10^{-03}$ & -50.6 & $1.64 \times 10^{-03}$ & 58.6 \\
SC $Q_{m} / Q_{t}$ & $4.88 \times 10^{-03}$ & -4.8 & $2.39 \times 10^{-03}$ & -6.1 & $2.50 \times 10^{-03}$ & 16.1 \\
SC & $3.80 \times 10^{-03}$ & -26.0 & $2.12 \times 10^{-03}$ & -16.7 & $2.54 \times 10^{-03}$ & 21.8 \\
SCI & $3.21 \times 10^{-03}$ & -37.4 & $1.94 \times 10^{-03}$ & -23.8 & $2.35 \times 10^{-03}$ & 33.1 \\
\hline
\end{tabular}

Note: ' $R$ ' represents the range, ' $S$ ' the straggling and ' $B$ ' the electrons backscattered to the cathode. The top section shows the results by assuming an isotropic DCS for the electrons with the elastic $Q_{m}$ from ZB. The bottom section shows the results by using the elastic DCS calculated by ZB. The labels are defined in table 1.

Table 4. Results for a scale factor of 10 and 100 and an input energy of $50 \mathrm{eV}$.

\begin{tabular}{llclrll}
\hline Simulation & $R_{z}(\mathrm{~m})$ & error $(\%)$ & $S_{z}(\mathrm{~m})$ & error $(\%)$ & $R_{x}(\mathrm{~m})$ & $S_{x}(\mathrm{~m})$ \\
\hline ZB & $6.62 \times 10^{-03}$ & 0.0 & $4.26 \times 10^{-03}$ & 0.0 & $-1.47 \times 10^{-07}$ & $5.04 \times 10^{-03}$ \\
FOR & $6.78 \times 10^{-03}$ & 2.5 & $4.33 \times 10^{-03}$ & 1.5 & $-3.77 \times 10^{-07}$ & $5.08 \times 10^{-03}$ \\
ISO & $6.02 \times 10^{-03}$ & -9.0 & $4.04 \times 10^{-03}$ & -5.3 & $-8.88 \times 10^{-07}$ & $4.90 \times 10^{-03}$ \\
\hline \multicolumn{7}{c}{ Scale factor 10} \\
\hline ZB & $1.62 \times 10^{-03}$ & 0.0 & $1.04 \times 10^{-03}$ & 0.0 & $1.46 \times 10^{-07}$ & $9.61 \times 10^{-04}$ \\
FOR & $1.87 \times 10^{-03}$ & 15.5 & $1.12 \times 10^{-03}$ & 7.6 & $-1.44 \times 10^{-07}$ & $8.89 \times 10^{-04}$ \\
ISO & $1.11 \times 10^{-03}$ & -31.4 & $8.40 \times 10^{-04}$ & -19.6 & $-1.15 \times 10^{-07}$ & $9.71 \times 10^{-04}$ \\
\hline
\end{tabular}

Note: ' $R$ ' represents the range and ' $S$ ' the straggling for a specific coordinate. The definitions of the abbreviations for the simulations are given in the text.

the probability for an inelastic process is low. The ISO model produces the largest relative error with $9 \%$. The errors for the FOR model is in the order of $2-3 \%$. The usage of forward scattering as an approximation for inelastic processes thus appears to be very accurate. For a scale factor of 100 the differences in results from the various models are more pronounced. Again the ISO model has the largest relative error with $31.4 \%$. The FOR model reaches a relative error of $15.5 \%$. The relative errors for predicting the range for a beam of $50 \mathrm{eV}$ are similar to the errors for the $200 \mathrm{eV}$ beam. The relative error of the predicted straggling is about $5 \%$ larger for the $50 \mathrm{eV}$ beam for a scale factor of 10 . The differences between the predictions for the beams are thus relatively small.

\subsection{Extrapolating the cross sections}

The elastic and inelastic optically allowed total cross sections can be extrapolated with

$$
Q_{t}(\epsilon)=c_{1} \frac{\ln \epsilon+c_{2}}{\epsilon},
$$

which is based on a simplified Bethe formula for excitation cross sections [33, equation (10)]. The high energy part of the momentum transfer cross section can be extrapolated with [7, equation (A.2)]

$$
Q_{m}=\frac{s_{1}}{\left(\epsilon-s_{2}\right)^{2}},
$$

with $c_{i}$ and $s_{i}$ fit parameters. The limit of the ratio of both expressions gives

$$
\lim _{\epsilon \rightarrow \infty} \frac{Q_{m}}{Q_{t}}=\lim _{\epsilon \rightarrow \infty} C \frac{\ln \epsilon}{\epsilon}=0 .
$$

Using this result in combination with (11) or (20) shows that the angular distributions for higher energies are approaching forward scattering. It is therefore expected that forward scattering is also a good approximation at energies above $200 \mathrm{eV}$. 


\section{Conclusions}

Analytical formulae for the DCS have been compared with the DCS calculated by Zatsarinny and Bartschat who used the B-spline R-matrix method for elastic and inelastic collisions for electrons with argon. The forward scattering of the elastic DCS can be approximated accurately by the screened Coulomb model that uses the parameterization given in equation (10). However the large angle behavior is not taken into account which makes the overall agreement rather poor. The inelastic DCS can not be accurately captured by any of the formulae that have been considered. Due to the lack of good analytical formulae direct fits of the DCS using equation (27) have been considered as well.

The degree of anisotropy has been quantified by comparing the range, straggling and backscattering of the various DCS models in a Monte Carlo simulation. An imaginary atom has been considered were only one elastic and one inelastic process is possible. The excitation process towards $4 s^{\prime}[1 / 2]_{1}$ is scaled by a factor 10 to approximately represent the total inelastic cross section of Ar. The simulations indicate that the DCS from the calculations of Zatsarinny and Bartschat can be approximated accurately. Errors in the order of one percent are obtained by using a direct fit of the DCS or an analytical fit using the ratio of $\frac{Q_{m}}{Q_{t}}$. For a scale factor of 10 for the inelastic cross section the errors using pure forward scattering are in the order of a 2-3\% which makes the forward model almost as accurate as a direct fit. Additionally, replacing the isotropic elastic scattering by the DCS calculated by Zatsarinny and Bartschat only modifies the results up to $2 \%$. This confirms that elastic scattering is accurately represented by the momentum transfer cross section from Zatsarinny and Bartschat and assuming isotropic scattering. It should be emphasized that the total elastic cross section and the elastic momentum transfer cross section are different, as shown in figure 5. By using scale factors for the inelastic process of 100 the total inelastic cross section is overestimated by a factor 10 approximately. The relative errors for the direct fit and the $\frac{Q_{m}}{Q_{t}}$-method are in the order of $0-7 \%$. The forward model gives relative errors of 7-15\%. Based on the considered tests the following recommendations are given

- For inelastic collisions, assume forward scattering (i.e. do not change the velocity angle)

- For elastic collisions, use the momentum-transfer cross section $Q_{m}$ (rather than the total cross section) for the collision probability, combined with an isotropic scattering angle from equation (22).

- This simple treatment performs better than any of the other theoretical/empirical analytical formulae for the range of conditions considered in our analysis, and we expect it to perform at least as well for general low-temperature plasma modeling purposes. The only way to obtain more accurate results is to use the full DCS, provided this is available.

There are a few caveats concerning these recommendations. The first is that our analysis did not take into account ionization because the data set from Zatsarinny and Bartschat does not include the ionization DCS. Ionization involves the additional question of how energy and momentum are shared between the primary and secondary electrons, a question that is not directly related to the anisotropy issue but can be equally important [13]. Secondly, in some molecular gases, unlike the argon-like gas in our analysis, angular velocity scattering by low-threshold rotational and vibrational processes can contribute significantly to the overall electron momentum losses. We could imagine that treating such low-threshold inelastic processes with forward scattering has consequences for the electron swarm parameters [34].

Previous works [6, 7] suggest that anisotropic scattering effects on swarm parameters are generally small. Calculations by Hagelaar of swarm parameters in Ar using cross sections from Zatsarinny and Bartschat [31] have been made for two types of scattering models (forward and isotropic) and two types of energy sharing models for ionization (50/50, the energy is split evenly between the electrons; $100 / 0$ the secondary electron gets no energy). A more realistic description of the energy redistribution [35] yields results intermediate between the two limiting cases $50 / 50$ and $100 / 0$.

The calculations show that changing the DCS for the inelastic processes from isotropic to forward scattering results in relative differences larger than $1 \%$ for $E / N$ values above $100 \mathrm{Td}$ for the mobility, average energy and the ionization rate. For the transverse and longitudinal diffusion coefficients differences of the order of $1 \%$ are already observed for $10 \mathrm{Td}$. At $1000 \mathrm{Td}$ the relative differences increased up to $29-32 \%$ for the mobility $\mu \mathrm{N}$. The impact of the energy sharing models is shown to be of the order of $1-9 \%$. For the ionization rate coefficient the impact of the anisotropy at $1000 \mathrm{Td}$ appeared to be $21-24 \%$ while the effect of the energy sharing model was smaller with $1-10 \%$. For the mean energy at $1000 \mathrm{Td}$ the effect of both models is smaller with the inelastic scattering models changing the results in the range of $13-15 \%$ and the energy sharing models changing the results only with $1-2 \%$. The longitudinal diffusion coefficient shows relative differences up to $100 \%$ at $1000 \mathrm{Td}$ induced by the anisotropy. The energy sharing model induces differences up to $35 \%$ at $250 \mathrm{Td}$. These results confirm that anisotropy is also important for swarm parameters and that over the $E / N$ range considered (10-1000 Td) its impact is comparable to the impact of the energy sharing models.

A more detailed study, that includes low-energy threshold processes, is required to evaluate the effect of anisotropy on swarm parameters relative to other assumptions in swarm calculations related to how electron number changing processes are taken into account.

\section{Acknowledgments}

The authors gratefully acknowledge support from PHC Van Gogh 2013, project $\mathrm{N}^{\circ}$ 28979ZE and the combined STWDraka project (10744). 


\section{References}

[1] Boeuf J P and Marode E 1982 J. Phys. D: Appl. Phys. 152169

[2] Kushner M J 1987 J. Appl. Phys. 61 2784-94

[3] Surendra M, Graves D B and Jellum G M 1990 Phys. Rev. A 41 1112-25

[4] Belenguer P and Pitchford L C 1999 J. Appl. Phys. 86 4780-5

[5] Petrović Z L, Dujko S, Marić D, Malović G, Nikitović Ž, Sašić O, Jovanović J, Stojanović V and Radmilović-Rađenović M 2009 J. Phys. D: Appl. Phys. 42194002

[6] Haddad G N, Lin S L and Robson R E 1981 Aust. J. Phys. 34243

[7] Phelps A V and Pitchford L C 1985 Phys. Rev. A 31 2932-49

[8] Thomas R W L and Thomas W R L 1969 J. Phys. B: At. Mol. Phys. 2562

[9] Reid I D and Hunter S R 1979 Aust. J. Phys. 32 255-60

[10] Kunhardt E E and Tzeng Y 1986 Phys. Rev. A 34 2158-66

[11] Stojanović V D and Petrović Z L 1998 J. Phys. D: Appl. Phys. 31834

[12] Hagelaar G J M and Pitchford L C 2005 Plasma Sources Sci. Technol. 14722

[13] Yoshida S, Phelps A V and Pitchford L C 1983 Phys. Rev. A 27 2858-67

[14] Zatsarinny O and Bartschat K 2004 J. Phys. B: At. Mol. Opt. Phys. 374693

[15] BSR database, Data type: differential scattering cross sections www.lxcat.net retrieved november 2013

[16] Allan M, Zatsarinny O and Bartschat K 2006 Phys. Rev. A 74030701

[17] Okhrimovskyy A, Bogaerts A and Gijbels R 2002 Phys. Rev. E 65037402

[18] Khrabrov A V and Kaganovich I D 2012 Phys. Plasmas 19093511
[19] Strickland D J, Book D L, Coffey T P and Fedder J A 1976 J. Geophys. Res. $812755-64$

[20] Mott N F and Massey H S W 1965 The Theory of Atomic Collisions (Oxford: Oxford University Press)

[21] Jacob J H 1973 Phys. Rev. A 8 226-35

[22] Hagelaar G 2000 Modeling of microdischarges for display technology PhD Thesis TUe (http://repository.tue. $\mathrm{nl} / 538700)$

[23] Brok W J M 2005 Modelling of transient phenomena in gas discharges PhD Thesis TUe (http://repository.tue.nl/595133)

[24] Laricchiuta A et al 2009 Eur. Phys. J. D 54 607-12

[25] Hayashi M 1981 Technical Report No. ippj-am-19 Institute of Plasma Physics, Nagoya University

[26] Massey H and Burhop E 1969 Electronic and Ionic Impact Phenomena (Oxford: Oxford University Press)

[27] Hagelaar G J M and Kroesen G M W 2000 Plasma Sources Sci. Technol. 9605

[28] White M D, Ross K J and Bolouri H 1979 J. Phys. B: At. Mol. Phys. 12811

[29] Longo S and Capitelli M 1994 Plasma Chem. Plasma Process. $141-13$

[30] van Dijk J, Peerenboom K, Jimenez M, Mihailova D and van der Mullen J 2009 J. Phys. D: Appl. Phys. 42194012

[31] BSR database, Data type: scattering cross sections www.lxcat. net retrieved november 2013

[32] Ahn S and Fessler J 2003 Standard errors of mean, variance and standard deviation estimators eECS Department, The University of Michigan http://web.eecs.umich.edu/fessler/ papers/files/tr/stderr.pdf

[33] Vriens L and Smeets A H M 1980 Phys. Rev. A 22 940-51

[34] Bordage M C, Ségur P and Chouki A 1996 J. Appl. Phys. 80 1325-36

[35] Opal C B, Peterson W K and Beaty E C 1971 J. Chem. Phys. 55 4100-6 